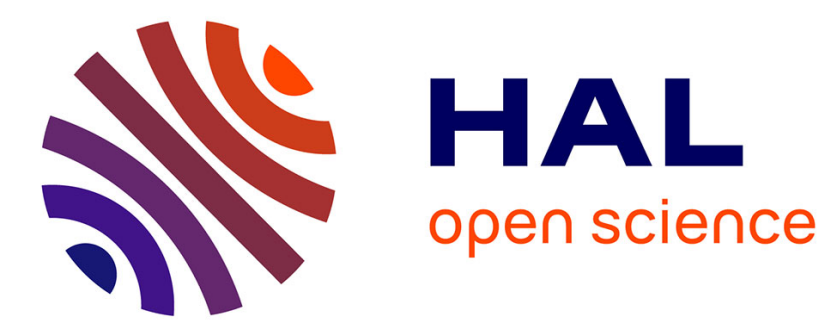

\title{
Diastereoselective Ring Homologation of Bicyclic Hydrazines: Access to cis -1,3-Diaminocyclohexitols
} Aurélie Blond, Serge Turcaud, Thomas Lecourt, Laurent Micouin

\section{To cite this version:}

Aurélie Blond, Serge Turcaud, Thomas Lecourt, Laurent Micouin. Diastereoselective Ring Homologation of Bicyclic Hydrazines: Access to cis -1,3-Diaminocyclohexitols. ACS Omega, 2018, 3 (11), pp.15302-15307. 10.1021/acsomega.8b02910 . hal-02186751

\section{HAL Id: hal-02186751 \\ https://hal.science/hal-02186751}

Submitted on 17 Jul 2019

HAL is a multi-disciplinary open access archive for the deposit and dissemination of scientific research documents, whether they are published or not. The documents may come from teaching and research institutions in France or abroad, or from public or private research centers.
L'archive ouverte pluridisciplinaire HAL, est destinée au dépôt et à la diffusion de documents scientifiques de niveau recherche, publiés ou non, émanant des établissements d'enseignement et de recherche français ou étrangers, des laboratoires publics ou privés. 


\title{
Diastereoselective Ring Homologation of Bicyclic Hydrazines: Access to cis-1,3-Diaminocyclohexitols
}

\author{
Aurélie Blond, ${ }^{\dagger}$ Serge Turcaud, ${ }^{\dagger}$ Thomas Lecourt, $^{\ddagger}{ }^{\dagger}$ and Laurent Micouin* ${ }^{\dagger}{ }^{\dagger}$ (i) \\ ${ }^{\dagger}$ Laboratoire de Chimie et de Biochimie Pharmacologiques et Toxicologiques, Faculté des Sciences Fondamentales et Biomédicales, \\ UMR 8601, CNRS-Paris Descartes University, 45 rue des Saints Pères, Paris 75006, France \\ ${ }^{\ddagger}$ Normandie Université, INSA Rouen, UNIROUEN, CNRS, COBRA UMR 6014, Rouen 76000, France
}

Supporting Information

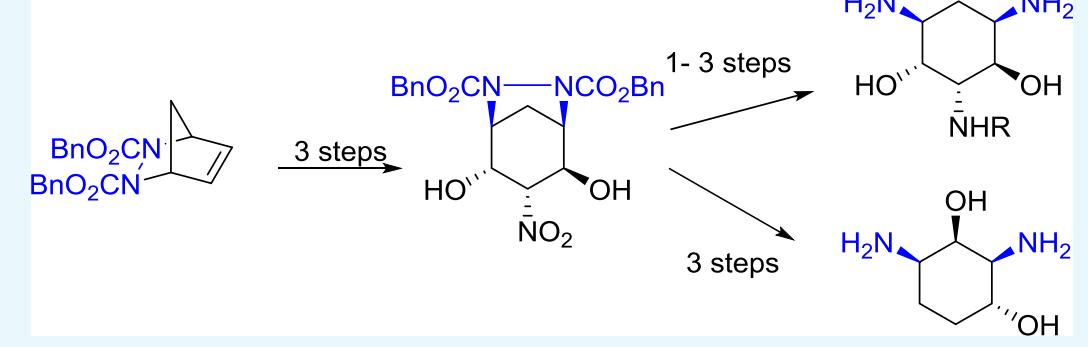

ABSTRACT: A sequence of oxidative cleavage/double nitroaldol condensation followed by a few simple synthetic transformations can lead to polyhydroxylated di- and triaminocyclohexanes from a readily available bicyclic hydrazine. This new synthetic route provides a simple and general access to densely substituted privileged scaffolds or fragments with a perfect control of their relative configuration.

\section{INTRODUCTION}

Aminocyclohexitols are a group of natural or synthetic compounds with numerous biological activities. ${ }^{1}$ Among them, cis-1,3-diaminocyclitols such as streptamine $\mathbf{1}$ or 2deoxystreptamine $\mathbf{2}$ are known to play a crucial role in the biological activity of aminoglycosides, a major class of antibiotics (Figure 1). ${ }^{2}$ Furthermore, substituted cis-1,3-



Figure 1. Examples of cis-1,3-diaminocyclitols and structural mimetics.

diaminocycloalkanes have been reported to be valuable fragments or scaffolds for the design of RNA binders. ${ }^{3}$ In this context, our group has been involved in the synthesis of polysubstituted 1,3-diamino cyclopentanes $3^{4}$ or piperidines $4^{5}$ starting from bicyclic hydrazine 5. ${ }^{6}$ Herein, we wish to report a straightforward and fully stereoselective approach toward hydroxylated 1,3-di- and 1,3,5-triaminocyclohexanes from a single precursor.

In our previous work, we have shown that aldehyde 6 could be obtained from hydrazine 5 using either ozonolysis or a twostep dihydroxylation-oxidative cleavage sequence. ${ }^{5}$ This aldehyde was then engaged in a reductive amination to deliver precursors of piperidines 4 . We envisaged the possibility of conducting a double nitroaldol reaction, from the same precursor 6, to prepare a bicyclic adduct 7 that would deliver polyhydroxylated 1,3,5-triaminocyclohexanes 8 after reductive cleavage of the hydrazine moiety (Scheme 1).

Cyclization of bis-aldehydes with nitromethane was indeed intensively investigated by Lichtenthaler and Ogawa on various substrates, including 1,5-dialdehydes derived from mono- or polysaccharides ("Sugar dialdehydes"), leading to diastereomeric mixtures of nitroinositols.

Such a strategy was also reported by Hasegawa and Sable for the preparation of triaminocyclohexanediols. ${ }^{8}$ From compound 9, obtained in seven steps from cis-3,5-dibromocyclopentene, reaction with nitromethane under basic conditions gave an equimolar amount of diastereoisomers $\mathbf{1 0}$ and $\mathbf{1 1}$ in $80 \%$ overall yield (Scheme 2).

\section{RESULTS AND DISCUSSION}

The nitroaldol condensation was investigated using compound 12, prepared from 5 in $86 \%$ yield, as the starting material (Table 1). The transient bis-aldehyde was generated by an oxidative cleavage and engaged in the next step without any other treatment than a filtration of the reaction mixture. No reaction was observed using sodium hydroxide ${ }^{9}$ as a base (entries 1-3). The use of sodium alkoxide ${ }^{10}$ led to the desired compound, albeit with a large amount of degradation product

Received: October 23, 2018

Accepted: November 1, 2018

Published: November 12, 2018 


\section{Scheme 1. Retrosynthetic Route}

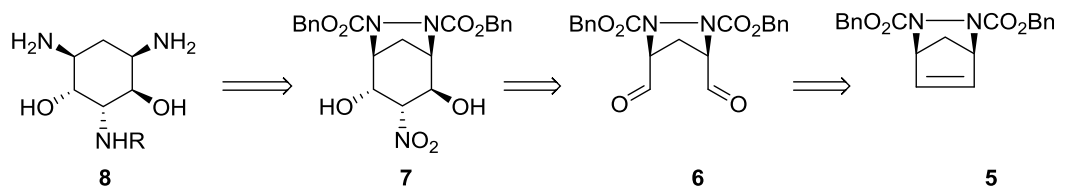

Scheme 2. Nitroaldol Condensation from 1,3Diaminocyclopentanediol 9

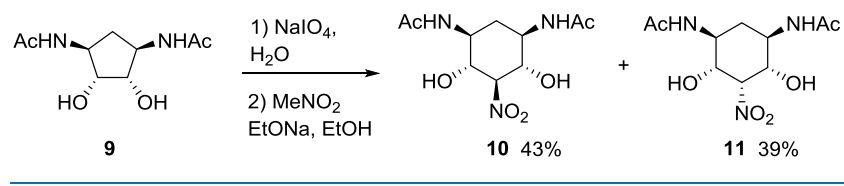

Table 1. Nitroaldol Reaction Optimization

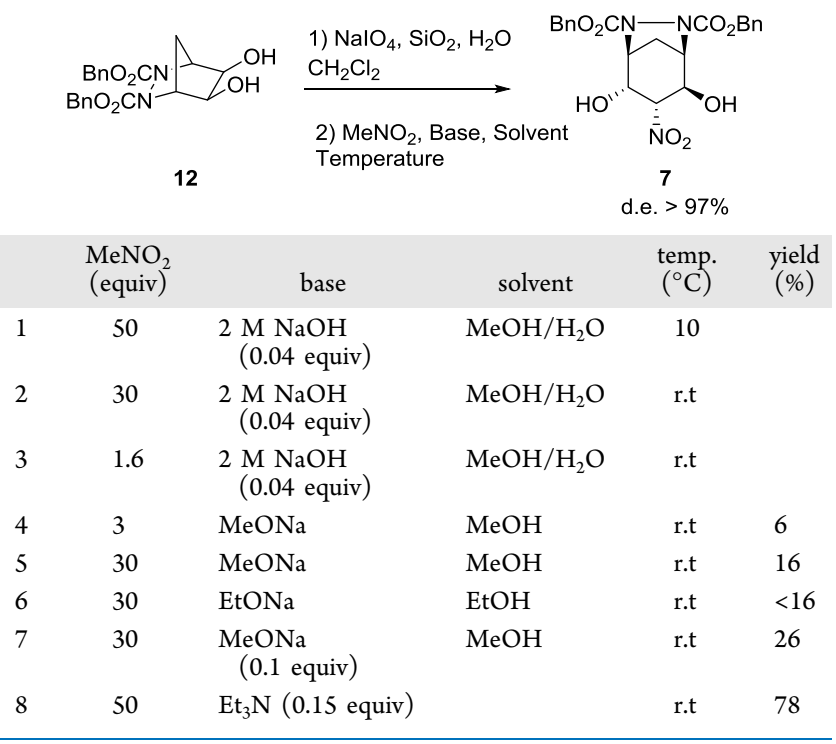

(entries 4-6). The presence of benzyl alcohol in the crude reaction mixture indicated that benzylcarbamates were cleaved under these basic conditions. A slight yield improvement could be obtained by lowering the base concentration (entry 7). Finally, best conditions were obtained using triethylamine ${ }^{11}$ as a base and running the reaction in nitromethane as a solvent (entry 8 ), leading to compound 7 in $78 \%$ yield on a multigram scale.

Although six possible diastereomers can theoretically be obtained with this condensation (Figure 2), compound 7 was formed as a single diastereomer, as determined by the NMR analysis of the crude reaction product. Its configuration could be determined by the NMR analysis of the purified compound. ${ }^{1} \mathrm{H}$ and ${ }^{13} \mathrm{C}$ NMR experiments revealed the absence of any plane of symmetry, discarding the formation of meso compounds. The equatorial orientation of the nitro group was furthermore established using correlation spectroscopy and nuclear Overhauser effect spectroscopy experiments. The stereoselective formation of 7 can be explained by the reaction of the transient bis-aldehyde keeping a $C_{s}$ symmetrical conformation, followed by ring closure and equilibration toward the thermodynamically favored bicyclic equatorial diastereomer. This thermodynamic control is much more efficient in the case of the formation of the rigid diazabicyclo[3.2.1] octane skeleton than with the synthesis of polysubstituted cyclohexanes reported by Hasegawa and Sable.

The reactivity of adduct 7 was then explored (Scheme 3). Hydrogenolysis under acidic conditions led to the triaminocyclohexitol $8 \mathbf{a}$ in a quantitative yield. The selective reduction of the nitro group was performed in the presence of zinc in acetic acid, leading to compound $\mathbf{1 3}$ in a quantitative yield. This compound could be functionalized by reductive amination with aldehyde or ketone, leading to compounds $\mathbf{1 4}$ and 15. N-substituted triaminocyclohexitols $\mathbf{8 b}$ and $\mathbf{8 c}$ were obtained after hydrogenolysis.

On the basis of our previous experience in acid-catalyzed stereoselective skeletal rearrangements of bicyclic hydrazines, we investigated the behavior of compound 13 under nitrous acid deamination conditions. ${ }^{12}$ Despite many experimental efforts, we were not able to obtain the corresponding alcohol from the diazonium intermediate. However, we were pleased to obtain compound $\mathbf{1 6}$ by adding a bromide source in the reaction mixture. The formation of 16 , established by NMR analysis, can be explained by the formation of a transient azetidinium followed by a selective ring opening, leading to rearranged compound $\mathbf{1 6}$ (Scheme 4$).^{13}$

Compound $\mathbf{1 6}$ was quantitatively converted into diamine $\mathbf{1 7}$ by hydrogenolysis (Scheme 5). ${ }^{14}$

\section{CONCLUSIONS}

In conclusion, we have reported a ring-homologation reaction of bicyclic hydrazines based on a sequential oxidative cleavage/

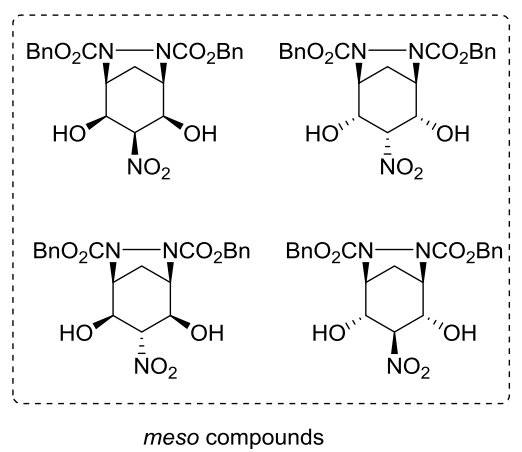

meso compounds

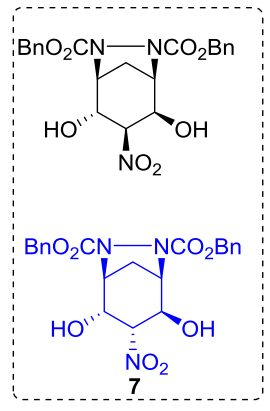

racemic compounds
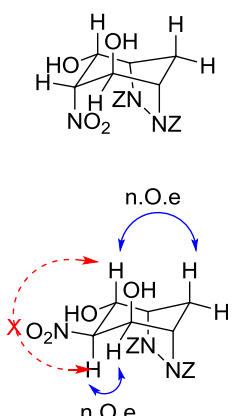

Figure 2. Possible stereoisomers and relative configuration of compound 7 . 
Scheme 3. Synthesis of cis-1,3-Diamino 5-Amino Cyclohexitols 8a-c

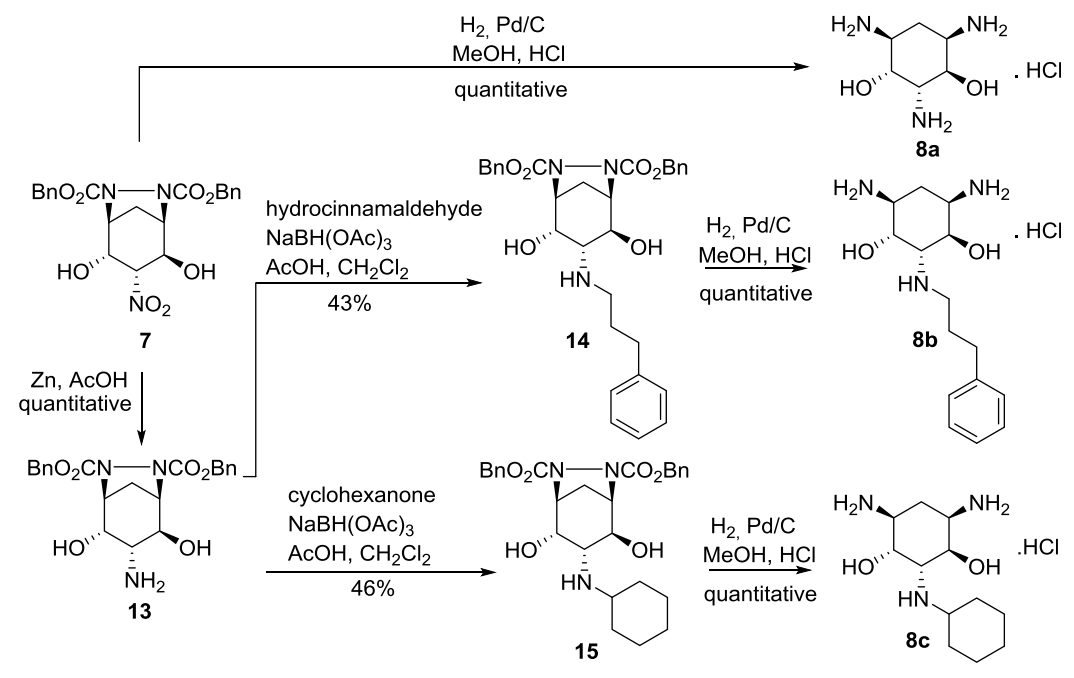

\section{Scheme 4. Synthesis of Compound 16}

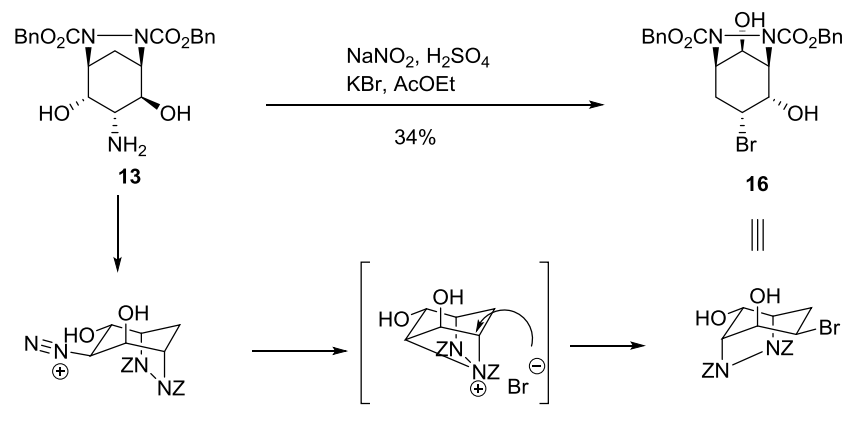

Scheme 5. Synthesis of cis-1,3-Diamino Cyclohexitol 17

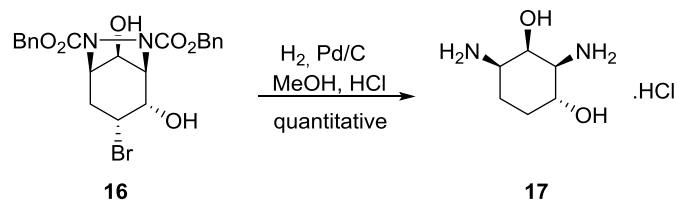

double nitroaldol condensation. This fully diastereoselective reaction enables the control of the relative configuration of five contiguous stereogenic centers in a single operation. The polyfunctionalized bicyclic hydrazine 7 , prepared on a gram scale from readily available bicyclic hydrazine 5 , furthermore provides a straightforward access to a large diversity of 1,3-diand 1,3,5-triaminocyclohexitols in 1-3 steps using simple synthetic transformations.

\section{EXPERIMENTAL SECTION}

All reagents and solvents were obtained from commercial suppliers and used as received without further purification. Compounds 5 and 12 were prepared according to reported procedures. ${ }^{5}$ Reactions were monitored by thin-layer chromatography (TLC) carried out on $0.20 \mathrm{~mm}$ silica gel (60-F254) with visualization by UV light or staining with phosphomolybdic acid, ninhydrin, or Dragendorff's reagent. Column chromatography was performed on silica gel $60 \AA$ (40-63 $\mu \mathrm{m})$. All ${ }^{1} \mathrm{H}$ NMR and ${ }^{13} \mathrm{C}$ NMR spectra were recorded on Bruker NMR spectrometers operating at 300,400 , or $500 \mathrm{MHz}$ $\left({ }^{1} \mathrm{H}\right.$ value) and at 75 or $125 \mathrm{MHz}\left({ }^{13} \mathrm{C}\right.$ value), respectively.
HRMS spectra were recorded on an Orbitrap mass spectrometer or on an ESI-QTOF II instrument Shimadzu.

Dibenzyl 2,4-Dihydroxy-3-nitro-6, 7 diazabicyclo[3.2.1]octane-6,7-dicarboxylate 7. To a vigorously stirred suspension of chromatographic grade silica gel $(9.90 \mathrm{~g})$ in $\mathrm{CH}_{2} \mathrm{Cl}_{2}(156 \mathrm{~mL})$ was added a $0.65 \mathrm{M}$ aqueous solution of $\mathrm{NaIO}_{4}(9.95 \mathrm{~mL}, 6.47 \mathrm{mmol}, 1.4$ equiv) dropwise while stirring, whence a flaky suspension was formed. Diol 12 ( $1.84 \mathrm{~g}, 4.62 \mathrm{mmol}, 1$ equiv) in $\mathrm{CH}_{2} \mathrm{Cl}_{2}(150 \mathrm{~mL})$ was then added, and the reaction was monitored by TLC until disappearance of the initial product. The reaction mixture was filtered on a sintered glass packed with $\mathrm{Na}_{2} \mathrm{SO}_{4}$, concentrated, and dissolved in nitromethane $(12.5 \mathrm{~mL}, 231$ mmol, 50 equiv). Triethylamine (96 $\mu \mathrm{L}, 0.69 \mathrm{mmol}, 0.15$ equiv) was added. The solution was stirred for $3 \mathrm{~h}$ at room temperature and was then concentrated. Flash chromatography $\left(\mathrm{CH}_{2} \mathrm{Cl}_{2} / \mathrm{MeOH} 98: 2\right)$ afforded $7(1.64 \mathrm{~g}, 3.59 \mathrm{mmol}, 78 \%)$ as a colorless oil.

${ }^{1} \mathrm{H}$ NMR $\left(300 \mathrm{MHz},\left(\mathrm{CD}_{3}\right)_{2} \mathrm{SO}, 70{ }^{\circ} \mathrm{C}\right): \delta(\mathrm{ppm}) 1.80(\mathrm{dt}$, $J=5.5,12.4 \mathrm{~Hz}, 1 \mathrm{H}), 2.38(\mathrm{~d}, J=12.4 \mathrm{~Hz}, 1 \mathrm{H}), 4.18(\mathrm{dd}, J=$ $4.9,9.5 \mathrm{~Hz}, 1 \mathrm{H}), 4.30-4.42(\mathrm{~m}, 2 \mathrm{H}), 4.50(\mathrm{dd}, J=2.5,5.5 \mathrm{~Hz}$, $1 \mathrm{H}), 4.63$ (br s, 1H), 5.00-5.27 (m, 4H), 5.64 (br s, $1 \mathrm{H}), 5.95$ $(\mathrm{d}, J=5.2 \mathrm{~Hz}, 1 \mathrm{H}), 7.30-7.38(\mathrm{~m}, 10 \mathrm{H}) ;{ }^{13} \mathrm{C} \mathrm{NMR}(75 \mathrm{MHz}$, $\left.\left(\mathrm{CD}_{3}\right)_{2} \mathrm{SO}, 70{ }^{\circ} \mathrm{C}\right): \delta 27.3,59.5,62.9,66.1,66.8,67.0,67.3$, 89.1, 127.1, 127.3, 127.6, 128.0, 135.6, 135.7, 153.8, 156.9; IR $\left(\mathrm{CH}_{2} \mathrm{Cl}_{2}\right) \nu: 3429,1701,1557,1498,1455,1388,1301,1190$, 1150, 1113, 1068, 1018, 955, 755, $698 \mathrm{~cm}^{-1}$; HRMS (ESI +-TOF) $m / z$ : calcd for $\mathrm{C}_{22} \mathrm{H}_{23} \mathrm{~N}_{3} \mathrm{O}_{8} \mathrm{Na}[\mathrm{M}+\mathrm{Na}]^{+}, 480.1377$; found, 480.1380 .

2,4,6-Triaminocyclohexane-1,3-diol 8a. To a solution of 7 ( $47 \mathrm{mg}, 103 \mu \mathrm{mol}, 1$ equiv) in $\mathrm{MeOH} \cdot \mathrm{HCl} \mathrm{pH} 3(1.8 \mathrm{~mL})$ was added $10 \%$ palladium on activated charcoal $(23 \mathrm{mg}, 0.2$ equiv). The resulting black suspension was stirred at room temperature under hydrogen ( $1 \mathrm{~atm})$ for $17 \mathrm{~h}$ and then hydrogen was bubbled in the reaction mixture few seconds. After stirring for 4 additional hours, the reaction mixture was filtered through a fritted glass funnel packed with Celite. Celite was washed with a $\mathrm{CH}_{2} \mathrm{Cl}_{2} / \mathrm{MeOH}$ mixture (50:50). The filtrate was concentrated to afford the final triamine 8a hydrochloride (18 mg, $103 \mu \mathrm{mol}$, quantitative yield) as a colorless oil.

${ }^{1} \mathrm{H}$ NMR (400 MHz, $\mathrm{CD}_{3} \mathrm{OD}$, hydrochloride): $\delta$ (ppm) $1.65(\mathrm{ddd}, J=11.7,5.7,4.6 \mathrm{~Hz}, 1 \mathrm{H}), 2.30(\mathrm{~d}, J=11.7 \mathrm{~Hz}$, 
$1 \mathrm{H}), 2.70(\mathrm{dd}, J=9.2,4.6 \mathrm{~Hz}, 1 \mathrm{H}), 3.32(\mathrm{dd}, J=5.7,1.6 \mathrm{~Hz}$, $1 \mathrm{H}), 3.40(\mathrm{dd}, J=9.2,1.6 \mathrm{~Hz}, 1 \mathrm{H}), 3.48(\mathrm{t}, J=4.6 \mathrm{~Hz}, 1 \mathrm{H})$, $3.78(\mathrm{t}, J=4.6 \mathrm{~Hz}, 1 \mathrm{H}) ;{ }^{13} \mathrm{C}$ NMR $\left(100 \mathrm{MHz}, \mathrm{CD}_{3} \mathrm{OD}\right.$, hydrochloride): $\delta$ (ppm) 31.4, 55.0, 60.1, 60.6, 71.2, 75.9; HRMS (ESI+-TOF) $m / z$ : calcd for $\mathrm{C}_{6} \mathrm{H}_{16} \mathrm{~N}_{3} \mathrm{O}_{2}[\mathrm{M}+\mathrm{H}]^{+}$, 162.1237; found, 162.1244 .

4,6-Diamino-2-((3-phenylpropyl)amino)cyclohexane1,3-diol 8b. Compound $\mathbf{8 b}$ hydrochloride was obtained from 14 in a quantitative yield $(19 \mathrm{mg}$, colorless oil) using the same procedure as for compound $8 \mathbf{a}$.

${ }^{1} \mathrm{H}$ NMR (500 MHz, $\mathrm{CD}_{3} \mathrm{OD}$, hydrochloride): $\delta$ (ppm) $1.80-1.96(\mathrm{~m}, 2 \mathrm{H}), 1.96-2.08(\mathrm{~m}, 1 \mathrm{H}), 2.20$ (br s, $1 \mathrm{H})$; 2.52-2.89 (m, 2H), 3.24 (br s, $1 \mathrm{H}), 3.49(\mathrm{t}, J=9.3 \mathrm{~Hz}, 1 \mathrm{H})$, $3.77(\mathrm{~d}, J=10.1 \mathrm{~Hz}, 1 \mathrm{H}), 4.14(\mathrm{~d}, J=9.3 \mathrm{~Hz}, 1 \mathrm{H}), 4.21$ (br s, $1 \mathrm{H}), 7.03-7.36(\mathrm{~m}, 5 \mathrm{H}) ;{ }^{13} \mathrm{C} \mathrm{NMR}\left(125 \mathrm{MHz}, \mathrm{CD}_{3} \mathrm{OD}\right.$, hydrochloride): $\delta$ (ppm) 28.0, 31.8, 34.4, 48.4, 59.6, 62.5, 63.7, 63.5, 71.1, 127.1, 129.6, 129.8, 143.0; IR (MeOH) $\nu: 3366$, 3230, 2939, 2866, 1599, 1513, 1455, 1212, 1103, $1042 \mathrm{~cm}^{-1}$; MS (ES+) $m / z: 280[\mathrm{M}+\mathrm{H}]^{+}$.

4,6-Diamino-2-(cyclohexylamino)cyclohexane-1,3diol 8c. Compound 8c hydrochloride was obtained from 15 in a quantitative yield (18 $\mathrm{mg}$, colorless oil) using the same procedure as for compound $\mathbf{8 a}$.

${ }^{1} \mathrm{H}$ NMR (400 MHz, $\mathrm{CD}_{3} \mathrm{OD}$, hydrochloride): $\delta$ (ppm) $1.22-1.47(\mathrm{~m}, 3 \mathrm{H}), 1.47-1.67(\mathrm{~m}, 2 \mathrm{H}), 1.72(\mathrm{~d}, J=13.0 \mathrm{~Hz}$, $1 \mathrm{H}), 1.92(\mathrm{~d}, J=13.0 \mathrm{~Hz}, 2 \mathrm{H}), 2.11(\mathrm{q}, J=12.4 \mathrm{~Hz}, 1 \mathrm{H})$, 2.22-2.39 (m, 3H), 3.35-3.42 (m, $1 \mathrm{H}), 3.72(\mathrm{ddd}, J=12.4$, $11.1,4.1 \mathrm{~Hz}, 1 \mathrm{H}), 3.81(\mathrm{dd}, J=5.0,3.1 \mathrm{~Hz}, 1 \mathrm{H}), 4.07(\mathrm{dt}, J=$ $12.4,3.3 \mathrm{~Hz}, 1 \mathrm{H}), 4.31(\mathrm{dd}, J=11.1,5.0 \mathrm{~Hz}, 1 \mathrm{H}), 4.44(\mathrm{t}, J=$ $3.1 \mathrm{~Hz}, 1 \mathrm{H}) ;{ }^{13} \mathrm{C}$ NMR (75 MHz, $\mathrm{CD}_{3} \mathrm{OD}$, hydrochloride): $\delta$ (ppm) 24.3, 24.6, 26.0, 28.5, 46.6, 48.4, 59.0, 59.6, 64.5, 64.6; IR $(\mathrm{MeOH}) \nu: 3366,3230,2938,2866,1599,1514,1456$, 1212, 1103, 1083, $1042 \mathrm{~cm}^{-1}$; HRMS (ESI+-Orbitrap) $\mathrm{m} / \mathrm{z}$ : calcd for $\mathrm{C}_{12} \mathrm{H}_{26} \mathrm{~N}_{3} \mathrm{O}_{2}[\mathrm{M}+\mathrm{H}]^{+}, 244.2020$; found, 244.2014.

Dibenzyl 3-Amino-2,4-dihydroxy-6, 7 diazabicyclo[3.2.1]octane-6,7-dicarboxylate 13. To a solution of 7 (304 mg, $664 \mu \mathrm{mol}, 1$ equiv) in distilled acetic acid $(5.2 \mathrm{~mL})$ was added activated zinc dust $(869 \mathrm{mg}, 13.3$ mmol, 20 equiv). After stirring for $2 \mathrm{~h}$ at $60{ }^{\circ} \mathrm{C}$, the reaction was filtered through a fritted glass funnel packed with Clarcel and then through a nylon syringe filter $0.45 \mu \mathrm{m}$. The filtrate was concentrated to afford $\mathbf{1 3}$ (404 mg, triacetate, quantitative yield) as a yellow oil. Compound 13 was systematically accompanied by 3 equiv of acetic acid and was characterized and further used as such.

${ }^{1} \mathrm{H}$ NMR (300 MHz, $\mathrm{CD}_{3} \mathrm{OD}, 50{ }^{\circ} \mathrm{C}$, triacetate): $\delta$ (ppm) $1.72-1.88(\mathrm{~m}, 1 \mathrm{H}), 1.97\left(\right.$ br s, $\left.9 \mathrm{H}, 3 \mathrm{CH}_{3} \mathrm{CO}\right), 2.40(\mathrm{~d}, J=$ $12.0 \mathrm{~Hz}, 1 \mathrm{H}), 2.82-2.97(\mathrm{~m}, 1 \mathrm{H}), 3.69-3.82(\mathrm{~d}, J=9.8 \mathrm{~Hz}$, $1 \mathrm{H}), 4.34$ (br s, $1 \mathrm{H}) ; 4.45$ (br s, $1 \mathrm{H}), 4.54$ (br s, $1 \mathrm{H}), 5.03-$ $5.29(\mathrm{~m}, 4 \mathrm{H}), 7.33$ (br s, $10 \mathrm{H}) ;{ }^{13} \mathrm{C}$ NMR $\left(75 \mathrm{MHz}, \mathrm{CD}_{3} \mathrm{OD}\right.$, $50{ }^{\circ} \mathrm{C}$, triacetate $): \delta(\mathrm{ppm}) 22.2\left(\mathrm{COCH}_{3}\right), 29.6,55.6,61.8$, 64.4, 67.1, 69.7, 69.5, 72.3, 129.1, 129.2, 129.4, 129.4, 129.6, 129.7, 137.3, 156.8, 159.5, 179.2; IR (MeOD) $\nu: 3425,1714$, 1559, 1455, 1421, 1348, 1154, 750, $699 \mathrm{~cm}^{-1}$; HRMS (ESI +-TOF) $m / z$ : calcd for $\mathrm{C}_{22} \mathrm{H}_{25} \mathrm{~N}_{3} \mathrm{O}_{6} \mathrm{Na}[\mathrm{M}+\mathrm{Na}]^{+}, 450.1636$; found, 450.1645 .

Dibenzyl 2,4-Dihydroxy-3-((3-phenylpropyl)amino)6,7-diazabicyclo[3.2.1]octane-6,7-dicarboxylate 14. To a solution of $13(160 \mathrm{mg}$, triacetate, $263 \mu \mathrm{mol}, 1$ equiv) in $\mathrm{CH}_{2} \mathrm{Cl}_{2}(0.9 \mathrm{~mL})$ and $\mathrm{MeOH}(0.1 \mathrm{~mL})$ was added hydrocinnamaldehyde ( $34 \mu \mathrm{L}, 263 \mu \mathrm{mol}, 1$ equiv), followed by $\mathrm{NaBH}(\mathrm{OAc})_{3}(223 \mathrm{mg}, 1.05 \mathrm{mmol}, 4$ equiv) and acetic acid $(0.05 \mathrm{~mL})$. After stirring for $20 \mathrm{~h}$ at room temperature, the reaction was quenched with saturated aqueous $\mathrm{NaHCO}_{3}$ and extracted with $\mathrm{CH}_{2} \mathrm{Cl}_{2}$, and the combined organic layers were dried over $\mathrm{MgSO}_{4}$, filtered, and concentrated. Flash chromatography $\left(\mathrm{CH}_{2} \mathrm{Cl}_{2} / \mathrm{MeOH}\right.$ 93:7 then $\mathrm{CH}_{2} \mathrm{Cl}_{2} /$ $\left(\mathrm{MeOH} / \mathrm{NH}_{4} \mathrm{OH} 30 \%\right.$ aq 90:10) 93:7) afforded $14(61 \mathrm{mg}$, $111 \mu \mathrm{mol}, 43 \%)$ as a colorless oil.

${ }^{1} \mathrm{H}$ NMR $\left(400 \mathrm{MHz},\left(\mathrm{CD}_{3}\right)_{2} \mathrm{SO}, 70{ }^{\circ} \mathrm{C}\right): \delta$ (ppm) 1.59$1.73(\mathrm{~m}, 3 \mathrm{H}), 2.16-2.28(\mathrm{~m}, 2 \mathrm{H}), 2.34$ (br s, $1 \mathrm{H}), 2.52$ (br s, $1 \mathrm{H}), 2.54-2.67(\mathrm{~m}, 2 \mathrm{H}), 3.35$ (dd, $J=9.3,1.5 \mathrm{~Hz}, 1 \mathrm{H}), 4.03$ (br s, $1 \mathrm{H}), 4.32(\mathrm{t}, J=4.7 \mathrm{~Hz}, 1 \mathrm{H}) ; 4.35(\mathrm{dd}, J=5.3,1.5 \mathrm{~Hz}$, $1 \mathrm{H}), 5.01-5.21(\mathrm{~m}, 4 \mathrm{H}), 7.09-7.43(\mathrm{~m}, 15 \mathrm{H}) ;{ }^{13} \mathrm{C}$ NMR $(75$ $\left.\mathrm{MHz},\left(\mathrm{CD}_{3}\right)_{2} \mathrm{SO}, 70^{\circ} \mathrm{C}\right): \delta(\mathrm{ppm}) 28.7,31.9,33.3,46.8,60.5$, $61.0,63.4,64.3,67.4,67.8,72.0,126.0,127.9,128.2,128.3$, 128.4, 136.7, 142.6, 155.1, 158.0; HRMS (ESI+-TOF) $\mathrm{m} / z$ : calcd for $\mathrm{C}_{31} \mathrm{H}_{36} \mathrm{~N}_{3} \mathrm{O}_{6}[\mathrm{M}+\mathrm{H}]^{+}$, 546.2599; found, 546.2601.

Dibenzyl 3-(Cyclohexylamino)-2,4-dihydroxy-6,7diazabicyclo[3.2.1]octane-6,7-dicarboxylate 15. To a solution of $13(134 \mathrm{mg}$, triacetate, $220 \mu \mathrm{mol}, 1$ equiv) in $\mathrm{CH}_{2} \mathrm{Cl}_{2}(1.3 \mathrm{~mL})$ was added cyclohexanone $(23 \mu \mathrm{L}, 220 \mu \mathrm{mol}$, 1 equiv), followed by $\mathrm{NaBH}(\mathrm{OAc})_{3}(186 \mathrm{mg}, 880 \mu \mathrm{mol}, 4$ equiv) and acetic acid $(0.05 \mathrm{~mL})$. The solution was stirred for $4 \mathrm{~h}$ at room temperature, and additional $\mathrm{NaBH}(\mathrm{OAc})_{3}(93 \mathrm{mg}$, $440 \mu \mathrm{mol}, 2$ equiv) was added. After stirring for 3 additional hours, the reaction was quenched with saturated aqueous $\mathrm{NaHCO}_{3}$ and extracted with $\mathrm{CH}_{2} \mathrm{Cl}_{2}$, and the combined organic layers were dried over $\mathrm{MgSO}_{4}$, filtered, and concentrated. Flash chromatography $\left[\mathrm{CH}_{2} \mathrm{Cl}_{2} / \mathrm{MeOH}\right.$ 95:5 then $\mathrm{CH}_{2} \mathrm{Cl}_{2} /\left(\mathrm{MeOH} / \mathrm{NH}_{4} \mathrm{OH} 30 \%\right.$ aq 90:10) 93:7] afforded 15 (52 mg, $102 \mu \mathrm{mol}, 46 \%)$ as a yellow oil.

${ }^{1} \mathrm{H}$ NMR (400 MHz, $\left.\left(\mathrm{CD}_{3}\right)_{2} \mathrm{SO}, 70{ }^{\circ} \mathrm{C}\right): \delta$ (ppm) 0.86$1.08(\mathrm{~m}, 2 \mathrm{H}), 1.08-1.32(\mathrm{~m}, 3 \mathrm{H}), 1.53($ br s, $1 \mathrm{H}), 1.58-1.78$ $(\mathrm{m}, 5 \mathrm{H}), 2.21(\mathrm{~d}, J=11.9 \mathrm{~Hz}, 1 \mathrm{H}), 2.33-2.47(\mathrm{~m}, 2 \mathrm{H}), 3.31$ (d, $J=9.1 \mathrm{~Hz}, 1 \mathrm{H}), 3.99$ (br s, $1 \mathrm{H}), 4.32(\mathrm{t}, J=4.3 \mathrm{~Hz}, 1 \mathrm{H})$, $4.35(\mathrm{~d}, J=4.9 \mathrm{~Hz}, 1 \mathrm{H}), 5.00-5.25(\mathrm{~m}, 4 \mathrm{H}), 7.24-7.44(\mathrm{~m}$, $10 \mathrm{H}) ;{ }^{13} \mathrm{C}$ NMR $\left(75 \mathrm{MHz},\left(\mathrm{CD}_{3}\right)_{2} \mathrm{SO}, 70{ }^{\circ} \mathrm{C}\right): \delta=24.6,24.8$, 26.2, 28.7, 33.0, 34.5, 53.9, 57.8, 60.6, 63.3, 65.1, 67.4, 67.8, 72.1, 127.9, 128.1, 128.3, 128.4, 128.7, 128.8, 136.6, 136.7, 155.1, 157.9; IR $\left(\mathrm{CH}_{2} \mathrm{Cl}_{2}\right) \nu: 3413,2928,2854,1706,1498$, $1451,1392,1306,1220,1185,1147,1092,1054,1022,753$, $698 \mathrm{~cm}^{-1}$; HRMS (ESI+-Orbitrap) $m / z$ : calcd for $\mathrm{C}_{28} \mathrm{H}_{36} \mathrm{~N}_{3} \mathrm{O}_{6}$ $[\mathrm{M}+\mathrm{H}]^{+}, 510.2599$; found, 510.2584.

Dibenzyl 3-Bromo-2,8-dihydroxy-6,7diazabicyclo[3.2.1]octane-6,7-dicarboxylate 16. To a solution of $13(148 \mathrm{mg}$, triacetate, $244 \mu \mathrm{mol}, 1$ equiv) in 2.5 $\mathrm{M}$ solution of $\mathrm{H}_{2} \mathrm{SO}_{4}(2.5 \mathrm{~mL})$ and ethyl acetate $(1 \mathrm{~mL})$ were added $\mathrm{KBr}\left(102 \mathrm{mg}, 854 \mu \mathrm{mol}, 3.5\right.$ equiv) and then $\mathrm{NaNO}_{2}$ $(120 \mathrm{mg}, 1.73 \mathrm{mmol}, 7.1$ equiv) dissolved in water $(1.36 \mathrm{~mL})$ dropwise at $0{ }^{\circ} \mathrm{C}$. The reaction mixture was stirred for $1 \mathrm{~h}$, and a half and additional $\mathrm{KBr}(102 \mathrm{mg}, 854 \mu \mathrm{mol}, 3.5$ equiv $)$ and then $\mathrm{NaNO}_{2}(120 \mathrm{mg}, 1.73 \mathrm{mmol}, 7.1$ equiv) dissolved in water $(1.36 \mathrm{~mL})$ were added. After stirring $16 \mathrm{~h}$ at room temperature, the reaction mixture was extracted with ethyl acetate. The organic layers were combined, dried over $\mathrm{MgSO}_{4}$, filtered, and concentrated. Flash chromatography $\left(\mathrm{CH}_{2} \mathrm{Cl}_{2} /\right.$ $\mathrm{MeOH}$ 98:2) afforded 16 (41 mg, $83 \mu \mathrm{mol}, 34 \%)$ as an orange oil.

${ }^{1} \mathrm{H}$ NMR $\left(300 \mathrm{MHz},\left(\mathrm{CD}_{3}\right)_{2} \mathrm{SO}, 70{ }^{\circ} \mathrm{C}\right): \delta(\mathrm{ppm}) 2.04(\mathrm{t}, J$ $=12.7,1 \mathrm{H}), 2.24-2.43(\mathrm{~m}, 1 \mathrm{H}), 3.85$ (ddd, $J=12.7,6.3,3.9$ $\mathrm{Hz}, 1 \mathrm{H}), 4.02-4.09(\mathrm{~m}, 1 \mathrm{H}), 4.11(\mathrm{~d}, J=4.5 \mathrm{~Hz}, 1 \mathrm{H}), 4.26$ $(\mathrm{d}, J=5.1 \mathrm{~Hz}, 1 \mathrm{H}), 4.46($ br s, $1 \mathrm{H}), 5.09-5.22(\mathrm{~m}, 4 \mathrm{H})$, $7.28-7.40(\mathrm{~m}, 10 \mathrm{H}) ;{ }^{13} \mathrm{C}$ NMR $\left(75 \mathrm{MHz},\left(\mathrm{CD}_{3}\right)_{2} \mathrm{SO}, 70{ }^{\circ} \mathrm{C}\right)$ : $\delta(\mathrm{ppm}) 35.7,48.8,64.5,67.7,67.9,68.7,69.7,127.9,128.0$,

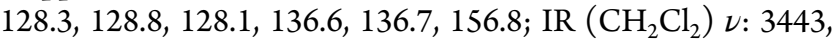


2927, 1704, 1498, 1456, 1391, 1328, 1296, 1213, 1149, 1102, 1062, 1022, 902, 755, $698 \mathrm{~cm}^{-1}$; HRMS (ESI+-TOF) $\mathrm{m} / z$ : calcd for $\mathrm{C}_{22} \mathrm{H}_{24} \mathrm{BrN}_{2} \mathrm{O}_{6} \mathrm{Na}[\mathrm{M}+\mathrm{Na}]^{+}, 513.0632$; found, 513.0634 .

2,4-Diaminocyclohexane-1,3-diol 17 . To a solution of $16(18 \mathrm{mg}, 37 \mu \mathrm{mol}, 1$ equiv) in $\mathrm{MeOH} \cdot \mathrm{HCl}(1 \mathrm{~mL})$ was added $10 \%$ palladium on activated charcoal $(10 \mathrm{mg}, 0.2$ equiv) under argon. The resulting black suspension was stirred at room temperature under hydrogen (1 atm) for $25 \mathrm{~h}$. $\mathrm{MeOH}$. $\mathrm{HCl} 1.25 \mathrm{M}(0.5 \mathrm{~mL})$ and $10 \%$ palladium on activated charcoal $(2 \mathrm{mg})$ were added under argon. The resulting black suspension was stirred at room temperature under hydrogen (1 atm) for $21 \mathrm{~h}$ and filtered through a nylon syringe filter 0.45 $\mu \mathrm{m}$. The filtrate was concentrated to afford the final diamine 17 hydrochloride (7 mg, quantitative yield) as an orange oil.

${ }^{1} \mathrm{H}$ NMR (400 MHz, hydrochloride, $\left.\mathrm{CD}_{3} \mathrm{OD}\right): \delta$ (ppm) $1.45-1.53(\mathrm{~m}, 1 \mathrm{H}), 1.78-1.90(\mathrm{~m}, 2 \mathrm{H}), 2.01-2.12(\mathrm{~m}, 1 \mathrm{H})$, $3.09(\mathrm{dd}, J=10.4,2.7,1 \mathrm{H}), 3.36-3.42(\mathrm{~m}, 1 \mathrm{H}) ; 3.81(\mathrm{td}, J=$ $11.0,4.9,1 \mathrm{H}), 4.29(\mathrm{t}, J=2.7,1 \mathrm{H}) ;{ }^{13} \mathrm{C} \mathrm{NMR}(75 \mathrm{MHz}$, hydrochloride, $\mathrm{CD}_{3} \mathrm{OD}$ ): $\delta$ (ppm) 22.2, 30.2, 51.0, 57.3, 65.3, 66.1; IR (MeOH) $\nu$ : 3351, 1697, 1642, 1422, 1370, 1238, $1100 \mathrm{~cm}^{-1}$; HRMS (ESI+-Orbitrap) $\mathrm{m} / z$ : calcd for $\mathrm{C}_{6} \mathrm{H}_{15} \mathrm{~N}_{2} \mathrm{O}_{2}[\mathrm{M}+\mathrm{H}]^{+}, 147.1128$; found, 147.1127.

\section{ASSOCIATED CONTENT}

\section{S Supporting Information}

The Supporting Information is available free of charge on the ACS Publications website at DOI: 10.1021/acsomega.8b02910.

$$
\text { Copies of }{ }^{1} \mathrm{H} \text { and }{ }^{13} \mathrm{C} \text { NMR spectra (PDF) }
$$

\section{AUTHOR INFORMATION}

\section{Corresponding Author}

*E-mail: laurent.micouin@parisdescartes.fr.

\section{ORCID}

Thomas Lecourt: 0000-0002-2532-3012

Laurent Micouin: 0000-0003-1080-8591

\section{Notes}

The authors declare no competing financial interest.

\section{ACKNOWLEDGMENTS}

Financial support from CNRS, MRT (grant to A.B.), is acknowledged.

\section{REFERENCES}

(1) (a) Duchek, J.; Adams, D. R.; Hudlicky, T. Chemoenzymatic Synthesis of Inositols, Conduritols, and Cyclitol Analogues. Chem. Rev. 2011, 111, 4223-4258. (b) Delgado, A. Recent Advances in the Chemistry of Aminocyclitols. Eur. J. Org. Chem. 2008, 3893-3906.

(2) Busscher, G. F.; Rutjes, F. P. J. T.; van Delft, F. L. 2Deoxystreptamine: Central Scaffold of Aminoglycoside Antibiotics. Chem. Rev. 2005, 105, 775-792.

(3) (a) Thomas, J. R.; Hergenrother, P. J. Targeting RNA with Small Molecules. Chem. Rev. 2008, 108, 1171-1224. (b) Carnevali, M.; Parsons, J.; Wyles, D. L.; Hermann, T. A Modular Approach to Synthetic RNA Binders of the Hepatitis C Virus Internal Ribosome Entry Site. ChemBioChem 2010, 11, 1364-1367. (c) Cottin, T.; Pyrkotis, C.; Stathakis, C. I.; Mavridis, I.; Katsoulis, I. A.; Anastasopoulou, P.; Kythreoti, G.; Zografos, A. L.; Nahmias, V. R.; Papakyriakou, A.; Vourloumis, D. Designed Spiro-Bicyclic Analogues Targeting the Ribosomal Decoding Center. ChemBioChem 2011, 12, 71-87. (d) Bodlenner, A.; Alix, A.; Weibel, J.-M.; Pale, P.; Ennifar, E.; Paillart, J.-C.; Walter, P.; Marquet, R.; Dumas, P. Synthesis of a
Neamine Dimer Targeting the Dimerization Initiation Site of HIV-1 RNA. Org. Lett. 2007, 9, 4415-4418. (e) Chung, F.; Tisné, C.; Lecourt, T.; Dardel, F.; Micouin, L. NMR-guided fragment-based approach for the design of tRNALys3 ligands. Angew. Chem., Int. Ed. 2007, 46, 4489-4491. (f) Moumné, R.; Pasco, M.; Prost, E.; Lecourt, T.; Micouin, L.; Tisné, C. Fluorinated Diaminocyclopentanes as Chiral Sensitive NMR Probes of RNA Structure. J. Am. Chem. Soc. 2010, 132, 13111-13113. (g) Lombès, T.; Moumné, R.; Larue, V.; Prost, E.; Catala, M.; Lecourt, T.; Dardel, F.; Micouin, L.; Tisné, C. Investigation of RNA-Ligand Interactions by $19 \mathrm{~F}$ NMR Spectroscopy Using Fluorinated Probes. Angew. Chem., Int. Ed. 2012, 51, 95309534.

(4) (a) Pérez Luna, A.; Ceschi, M.-A.; Bonin, M.; Micouin, L.; Husson, H.-P.; Gougeon, S.; Estenne-Bouhtou, G.; Marabout, B.; Sevrin, M.; George, P. Enantioselective Desymmetrization of Meso Bicyclic Hydrazines: A Novel Approach to the Asymmetric Synthesis of Polysubstituted Amino Cyclopentanic Cores. J. Org. Chem. 2002, 67, 3522-3524. (b) Bournaud, C.; Bonin, M.; Micouin, L. Skeletal Rearrangements in the 2,3-Diazanorbornene Series. A Fast Access to Highly Functionalized Cyclopentanes. Org. Lett. 2006, 8, 3041-3043. (c) Pasco, M.; Moumné, R.; Lecourt, T.; Micouin, L. Stereoselective Synthesis of Fluorinated 1,3-cis-Diaminocyclopentanes. J. Org. Chem. 2011, 76, 5137-5142.

(5) Blond, A.; Dockerty, P.; Álvarez, R.; Turcaud, S.; Lecourt, T.; Micouin, L. Modular Access to N-Substituted cis-3,5-Diaminopiperidines. J. Org. Chem. 2013, 78, 12236-12242.

(6) (a) Santhini, P. V.; Smrithy, A. S.; Irfana Jesin, C. P.; Varughese, S.; John, J.; Radhakrishnan, K. V. Accessing highly functionalized cyclopentanoids via a cascade palladation approach: unprecedented benzylic C-H activation towards cyclopentenoindanes. Chem. Commun. 2018, 54, 2982-2985 and references inside . (b) Bournaud, C.; Chung, F.; Perez Luna, A.; Pasco, M.; Errasti, G.; Lecourt, T.; Micouin, L. Stereoselective Transformations of meso Bicyclic Hydrazines: Versatile Access to Functionalized Aminocyclopentanes. Synthesis 2009, 869-887. (c) Sajisha, V. S.; Anas, S.; John, J.; Radhakrishnan, K. V. Desymmetrization of meso-Bicyclic Hydrazines: An Efficient Strategy towards the Synthesis of Functionalized Cyclopentenes. Synlett 2009, 2885.

(7) (a) Lichtenthaler, F. W. New Methods of Preparative Organic Chemistry IV. Cyclization of Dialdehydes with Nitromethane. Angew. Chem., Int. Ed. 1964, 3, 211-224. (b) Ogawa, S.; Orihara, M. Synthesis of seven penta-N,O-acetyl-pseudo-2-amino-2-deoxy-dlhexopyranoses. Carbohydr. Res. 1988, 177, 199-212.

(8) Hasegawa, A.; Sable, H. Z. Studies on cyclitols-XII. Tetrahedron 1969, 25, 3567-3578.

(9) Eberle, M.; Missbach, M.; Seebach, D. Enantioselective Saponification with Pig Liver Esterase (PLE): (1S,2S,3R)-3Hydroxy-2-nitrocyclohexyl Acetate. Org. Synth. 1990, 69, 19.

(10) Ogawa, S.; Aoyama, H.; Sato, T. Synthesis of an ether-linked alkyl 5a-carba- $\beta$-d-glucoside, a 5a-carba- $\beta$-d-galactoside, a 2 -acetamido-2-deoxy-5a-carba- $\beta$-d-glucoside, and an alkyl $5 \mathrm{a}^{\prime}$-carba- $\beta$-lactoside. Carbohydr. Res. 2002, 337, 1979-1992.

(11) Jain, A.; Rodríguez, S.; López, I.; González, F. V. Highly stereoselective epoxidation of O-protected 3-hydroxy-1-nitroalkenes. Tetrahedron 2009, 65, 8362-8366.

(12) Kolitz, M.; Cohen-Arazi, N.; Hagag, I.; Katzhendler, J.; Domb, A. J. Biodegradable Polyesters Derived from Amino Acids. Macromolecules 2009, 42, 4520-4530.

(13) (a) Couty, F.; Durrat, F.; Evano, G. Regioselective nucleophilic opening of azetidinium ions. Synlett 2005, 1666-1670. (b) Couty, F.; Evano, G. Azetidines: new tools for the synthesis of nitrogen heterocycles. Synlett 2009, 3053-3064. For a closely related rearrangement involving bicyclic hydrazones, see: Bournaud, C.; Bonin, M.; Micouin, L. Skeletal rearrangements in the 2,3Diazanorbornene Series. A Fast Access to Highly Functionalized Cyclopentanes. Org. Lett. 2006, 8, 3014-3043.

(14) For a closely related debromination under hydrogenolysis conditions, see: Boehme, W. R. Stereochemistry of Diels-Alder Adducts. III. The Preparation and Rearrangement of Some 
Brominated Derivatives of Norbornanecarboxylic Acids. J. Am. Chem.

Soc. 1959, 81, 2762-2765. 